\title{
ON THE CALCULATION OF TWO ESSENTIAL HARMONIC SERIES WITH A WEIGHT 5 STRUCTURE, INVOLVING HARMONIC NUMBERS OF THE TYPE $H_{2 n}$
}

\section{CORNEL IOAN VĂLEAN}

Abstract. The core of the present paper is represented by the calculation of two essential harmonic series with a weight 5 structure, involving harmonic numbers of the type $H_{2 n}$. The two main series are evaluated by also exploiting results and strategies presented in the book, (Almost) Impossible Integrals, Sums, and Series, 2019.

Mathematics subject classification (2010): 26A36, 33B30, 65B10, 40C10, 40G10, 11 M06.

Keywords and phrases: Harmonic numbers, harmonic series, logarithmic integrals, polylogarithm function, Riemann zeta function, Dirichlet eta function.

\section{REFERENCES}

[1] H.M. Srivastava, J. Choi, Series Associated with the Zeta and Related Functions, Springer (originally published by Kluwer), Dordrecht, 2001.

[2] C.I. VĂLEAN, (Almost) Impossible Integrals, Sums, and Series, Springer, New York, 2019.

[3] C.I. VĂLEAN, A master theorem of series and an evaluation of a cubic harmonic series, JCA 10, no.2, 97-107, 2017.

[4] C.I. VĂLEAN, A new powerful strategy of calculating a class of alternating Euler sums, https://www.researchgate.net/publication/333999069, 2019. 